\title{
Intervención psicológica en el ámbito hospitalario
}

\section{Psychological intervention in the hospital setting}

\author{
Ana M. López Fuentetaja ${ }^{(1)}$ y Odei Iriondo Villaverde ${ }^{(2)}$ \\ (1) Hospital Universitario 12 de Octubre. Madrid, España. \\ (2) CSMIJ L'Hospitalet de Llobregat - Fund. Orienta, España.
}

\begin{abstract}
Resumen: La atención psicológica en el ámbito hospitalario representa una modalidad de intervención de alta complejidad, en la que confluyen actividades asistenciales, docentes y de investigación. Se parte de una consideración bio-psico-social del paciente ingresado o enfermo, que incluye las características particulares del contexto y las diferentes dinámicas que forman parte de la institución hospitalaria. Para llevar a cabo sus funciones, el psicólogo clínico precisa conocer, adaptarse y construir progresivamente un lenguaje común con el resto de facultativos y profesionales sanitarios, pero sin perder lo específico de su propia especialidad formativa. Incluir y transmitir la perspectiva psicosomática en la comprensión del paciente será un objetivo prioritario de la intervención psicológica. La estructura organizativa y el lugar ocupado por el psicólogo dentro de la misma, fundamentalmente en unidades de interconsulta-enlace, junto a los recursos y medios con los que se cuenta, condicionarán las diferentes modalidades de intervención y el desarrollo de diferentes programas de enlace.

Palabras clave: institución hospitalaria, psicología clínica hospitalaria, psicosomática, interconsulta-enlace, programas de enlace, dificultades del psicólogo clínico.
\end{abstract}

\begin{abstract}
Psychological care in the hospital setting represents a highly complex type of intervention including care, teaching, and research activities. It is based on a biopsychosocial consideration of the hospitalized or sick patient, which includes the unique characteristics of the context and the different dynamics inherent to the hospital institution. To carry out their role, clinical psychologists need to know, adapt, and progressively build a common language with all other physicians and healthcare professionals, but without losing the specificity of their own specialty. Including and transmitting the psychosomatic perspective in patient understanding will be a priority objective of psychological intervention. The organizational structure and the place of the psychologist within it, mainly at interconsultation-liaison units,
\end{abstract}

\footnotetext{
Ana M. López Fuentetaja es psicóloga clínica en el Hospital Universitario 12 de Octubre, Madrid.

Odei Iriondo Villaverde es Psicóloga Clínica en el CSMIJ L’Hospitalet de Llobregat - Fund.Orienta.

La correspondencia sobre este artículo debe enviarse a los autores al e-mail: alofuen@gmail.com (Ana M. López-Fuentetaja);

odei.iriondo@gmail.com (Odei Iriondo).

(cc) EY-NC-ND Este es un artículo Open Access bajo la licencia CC BY-NC-ND.
} 
together with the available resources and means, will condition the different modalities of intervention and the development of different liaison programs.

Keywords: hospital institution, clinical hospital psychology, psychosomatic, interconsultation-liaison, liaison programs, difficulties of clinical psychologist.

\section{La institución hospitalaria}

“Mi humanidad está en sentir que somos voces de una misma penuria” J. L. Borges

\section{Organización y dinámicas}

Los hospitales, de forma complementaria con otros dispositivos, tienen como objetivo primordial la atención a la salud pública y la formación de nuevos profesionales especializados. Tras la organización de los mismos, encontramos una Administración, que define políticas y normativas y establece prioridades, delegando en gestores y profesionales la garantía de funcionamiento para que dichos objetivos puedan ser cumplidos. En este sentido, los diferentes profesionales no son totalmente autónomos, ya que están condicionados en sus actuaciones por la propia definición de dichas normativas y por su inclusión en equipos de trabajo, pero a la vez, deben ostentar un margen de autonomía, derivada de su propio rol, capacitación y opinión en el proceso de toma de decisiones.

Los propios miembros y equipos que forman parte de las instituciones generan fenómenos de interrelaciones y dinámicas que pueden fomentar la salud y la consecución de los objetivos para los que fueron creadas o, por el contrario, relegar estos a segundo plano, como consecuencia de esas dinámicas presentes en un momento determinado. Encontraríamos aquí un amplio número de fenómenos, que se dan en todas las relaciones humanas cargadas de fuerte emocionalidad, que si se anteponen a las condiciones que hacen posible el trabajo, supondrían una especie de boicot hacia el mismo, aunque no sea algo intencionado. Por nombrar algunos, encontraríamos entre ellos rivalidades, intereses personales que dirigen la priorización de tareas alejando estas de las necesidades de los pacientes, omnipotencia o impotencia desgastantes, como fruto del desajuste entre las exigencias y los medios con los que se cuenta, culpabilización del resto de profesionales cuando las cosas no funcionan bien, proyecciones, etc. Estos procesos que condicionan nuestro trabajo haciéndolo poco operativo, con frecuencia no pensados, solo los podremos contrarrestar, en la medida que podamos tener acceso al conocimiento de los mismos (Bion, 1974).

Precisamos, por tanto, de un marco formativo y un proceso reflexivo para su reconocimiento. De esta manera, la indagación sobre estos fenómenos y las tensiones producidas a consecuencia de los mismos, a las cuales también están sujetos los propios psicólogos, constituyen en sí un elemento que posibilita su modificación, ya que introducir pensamiento y comprensión permite actuar sobre dichas dinámicas.

Los estilos de liderazgo y el lugar y poder que desde la jerarquía se otorga a cada profesional o colectivo, los procesos de comunicación tanto a un nivel vertical como horizontal, junto a la articulación entre los medios con los que se cuenta y la realización de determinadas tareas encomendadas, como nombrábamos anteriormente, son elementos clave en la generación de tensiones, siendo este el campo específico en el que el psicólogo está inmerso y en que debe actuar (Bleger, 1994). Se trataría, por tanto, de comprender la situación para poder generar condiciones acordes a los objetivos de salud y desde esa comprensión intervenir y favorecer cambios.

La institución hospitalaria es, por tanto, una instancia que atraviesa lo individual (de cada profesional y de cada paciente), lo relacional (entre pacientes y profesionales, entre pacientes entre sí y profesionales entre sí, entre familiares y pacientes/profesionales), lo grupal (entre equipos y sub-equipos de un mismo servicio, entre servicios diferentes) y la propia organización (organigrama, jerarquía, liderazgo, funcionamiento, grado de flexibilidad, participación, etc.). Todos esos elementos están presentes e interaccionan en lo que llamamos institución, es decir, la constituyen, la definen, la van modificando a través del tiempo y producen unos deter- 
minados efectos en lo que podríamos llamar su objeto de trabajo. A su vez, la organización debe cumplir la función de ordenar el sistema para que los objetivos para los que fue constituida se cumplan.

Por otra parte, la institución hospitalaria, preexiste y cuenta con una historia previa cuando un nuevo miembro o colectivo se incorpora a ella, inscribiéndole en sus discursos y en sus vínculos (Kaës, 1989) y requiriendo para su plena pertenencia de un esfuerzo de adaptabilidad tanto por parte del que llega como del que ya estaba ahí. En dicha inscripción, pueden aparecer aspectos beneficiosos y otros que ya no lo son tanto. Estos últimos, por señalar algunos, los encontraríamos en actuaciones al servicio de intereses individuales, al margen de los objetivos o fines terapéuticos, toma de decisiones no reflexionadas, fenómenos relacionados con el ejercicio del poder, exagerada resistencia al cambio o compulsiones e inercia de los aspectos más burocráticos, etc.

Hay que tener en cuenta también que cada miembro de la institución precisa de una cierta armonía con la misma para que la colaboración sea posible y equilibrada, requiriendo para ello a su vez que esta sostenga el narcisismo de sus miembros para que no sea atacada o devaluada (Kaminsky, 1990).

La identidad institucional se define así por su presente y por su historia, yendo más allá de cualquier circunstancia inmediata de una persona o grupo y trascendiendo a su singular permanencia o ausencia. De esta forma, continuidad y cambio se producen de forma constante y simultánea, como fruto de la interacción de cada uno de esos profesionales y pacientes pertenecientes a la misma y de las circunstancias de cada momento, existiendo la posibilidad de una crisis en los momentos en que el cambio supera o rompe de forma abrupta el equilibrio previo. Por otra parte, los procesos de cambio institucionales, requieren intencionalidad y esfuerzo, y suelen producirse de forma muy lenta, siendo la inercia un singular motor de funcionamiento.

\section{Contexto hospitalario}

Las características del contexto hospitalario tienen una influencia tanto en los pacientes como en los propios profesionales, generando un impacto para ambos. Podríamos hablar del encuentro de dos desconocidos llamados a entenderse, pero que no tienen porqué encontrarse en las mejores condiciones para ello, sometidos ambos a sus propias circunstancias personales.

Aunque pueda parecer una obviedad, cabe explicitar que el hospital es un escenario en el que la experiencia del sufrimiento, tanto físico como psíquico, está constantemente presente, realidad que los diferentes profesionales sanitarios tienen que afrontar de manera excelente en su práctica habitual.

Si bien el dolor físico pertenece a la esfera de los pacientes, el dolor psíquico se expresa tanto en pacientes como en profesionales, ya que no es posible acercarse a un paciente que sufre sin que este sufrimiento encuentre un eco en el profesional mismo. La demanda imperiosa presente en nuestra cultura, por parte de pacientes y familiares, de alivio, curación y esperanza, pone a prueba las fortalezas de los profesionales que inevitablemente tienen que contactar con sus propias limitaciones, ya que la realidad impone que esa expectativa no siempre pueda ser conseguida.

Si bien la posición del paciente ingresado y los profesionales es claramente asimétrica, cada uno de ellos a su manera puede encontrar en el hospital un ambiente estresante.

Durante el ingreso hospitalario, las características de alejamiento o reducción de los apoyos sociales y ambientales habituales, así como la pérdida de intimidad, de individualidad y de control sobre las actividades cotidianas, son para los pacientes algunos de los primeros elementos productores de estrés (Groves y Muskin, 2006).

Estos elementos pueden favorecer en el paciente más vulnerable un desequilibrio significativo, que no le permita adoptar estrategias de afrontamiento eficaces. Para el enfermo, desprenderse de su ropa y objetos, someterse a pruebas invasivas, compartir habitaciones, soportar interrupciones del sueño para la realización de distintas actuaciones sobre él mismo o sobre otro enfermo o ruidos constantes en determinadas unidades de vigilancia, suponen un malestar añadido a la propia enfermedad.

Sin embargo, también hay otra serie de circunstancias generadoras de estrés o ansiedad que comparten tanto profesionales como pacientes. Entre ellas, podemos encontrar el factor de incertidumbre (Rodríguez y Zurriaga, 1997) sobre el pronóstico y evolución de determinadas enfermedades, sobre todo si consideramos que las propias intervenciones clínicas, con frecuencia, forman parte también de ese pronóstico. 
Igualmente, las dificultades de comunicación (Zas, 2011) pueden ser cuanto menos inquietantes para unos y otros. Si compartir información es complicado en sí, los procesos de comunicación se pueden dificultar aún más ante pacientes que por su situación de enfermedad se ven privados de la posibilidad de entender, hablar o escuchar, o también por la gran confluencia de personas y colectivos, con culturas y perspectivas diferentes. En el ámbito hospitalario, son numerosos los momentos en que compartir información resulta imprescindible, pudiendo representar la presencia de barreras idiomáticas un añadido de dificultad, tanto para el paciente como para el profesional. Por otra parte, aun compartiendo el mismo idioma, el argot médico puede producir incomprensión e incomunicación en el paciente, de la misma forma que la perspectiva y cultura del paciente, $\mathrm{o}$ su bloqueo ante determinadas (malas) noticias, pueden también producirlas en los profesionales.

Asimismo, se podría considerar como otra fuente de estrés para el profesional la interacción con pacientes insatisfechos y "difíciles", en los que son precisamente las características psicopatológicas y psicosociales las que dificultan la confianza y cooperación necesaria. Por otra parte, los propios profesionales tratantes, por características relativas a sus condiciones laborales, como pueden ser la inestabilidad, masificación, ubicación o carencia de medios, pueden también estar sometidos a importantes niveles de estrés o burnout (Gómez, 2004).

Las sensaciones desagradables que los profesionales no pueden elaborar, compartir o canalizar, se manifiestan en agotamiento, pérdida de control sobre sus actuaciones, bien por exceso o por defecto, falta de autoridad, disminución de su autoestima, temor, rechazo, frustración, enfado, etc., que pueden dificultar su tarea cotidiana, haciéndoles más insensibles hacia las necesidades de los pacientes.

Sin embargo, a pesar de lo señalado anteriormente, a la mayoría de pacientes y más cuanto más graves, el contexto hospitalario les ofrece posibilidades protectoras y esperanzadoras, viviéndolo como un lugar de cuidado. El deseo de mejora o curación y el sentirse cuidado y tratado, permite tolerar y sublimar el sufrimiento. En este sentido, tanto el trato recibido por parte de los profesionales, como una buena comunicación representan elementos fundamentales de satisfacción (Heyland, Rocker, Dodek y Kutsogiannis,2002; Ricart, 2008) más allá de la propia evolución de la enfermedad o mejoría de los síntomas, quedando grabadas las vivencias y la conexión emocional con los profesionales y con otros pacientes. También el propio ingreso o consulta, en ese espacio de especialistas en la materia, contribuye al sentimiento de confianza del paciente, sintiéndose en las mejores manos.

Por último, podríamos considerar que la llegada de un paciente a un dispositivo hospitalario es, a veces, la primera ocasión para que pueda recibir una atención clínica, social o preventiva, incluido en el ámbito de su salud mental, y que se inicie un proceso de tratamiento, siendo responsabilidad del profesional que esta ocasión sea detectada y atendida.

En todo este contexto, es fundamental tener presente la importancia de responder a las necesidades psicológicas, ya que suele ocurrir que a mayor gravedad médica, por la urgencia en las actuaciones, menor cuidado se tenga frente a aspectos psicológicos.

De forma complementaria, cuando hablamos de necesidades psicológicas, no solo nos referimos a las de los pacientes, sino también a las de los profesionales sanitarios, quienes pueden verse necesitados de asesoramiento sobre cómo abordar y manejar complejas situaciones, en las que los procesos psicoemocionales de ambos pueden desempeñar un papel nuclear y que trascienden el conocimiento y el saber hacer de sus respectivos campos de especialización, encontrándose por ello desprovistos de herramientas para su manejo efectivo.

\section{El Psicólogo Clínico en el Hospital}

\section{Reflexiones sobre algunos aspectos de especial dificultad}

Acercarse al cuerpo de los pacientes. La formación de la que partimos los psicólogos clínicos no está familiarizada con la mirada del cuerpo del otro. Mucho menos de un cuerpo enfermo, mutilado, dañado, envejecido, desnudo o conectado a respiradores, vías y diferentes máquinas de soporte. Lo que para otros profesionales ha sido el día a día de su formación, para nosotros supone un impacto importante. En ocasiones, el cuerpo 
enfermo pone en evidencia el olor de los diferentes fluidos corporales, produciendo diversas reacciones a las que no es fácil acostumbrarse. Esto también ocurre con mayor frecuencia en el interior de algunos dispositivos hospitalarios, como las unidades de reanimación, trasplantes, transición, UCIS. El paciente hospitalizado siente la necesidad de mostrar su cuerpo y que esa necesidad sea tolerada y acogida, al sentirlo como objeto prioritario de fragilidad y desvalimiento. Es importante tener en cuenta que es imposible que el paciente pueda identificarse con nosotros y con nuestro discurso, sin vivirlo como ajeno o invasivo, si no nos percibe a la vez como alguien identificado con su sufrimiento.

Tolerar la realidad de la vulnerabilidad y la muerte. La intervención psicológica con el paciente ingresado se realiza en tiempo real con el acontecimiento traumático, la situación de urgencia, el fracaso terapéutico o el mal pronóstico vital. El relato del paciente es muy diferente al que ocurre en el marco de una consulta ambulatoria, en la que el paciente narra lo ocurrido y el hecho es traído y representado, pudiendo trabajar con mayor facilidad con elementos simbólicos. En el ámbito del paciente ingresado, de alguna forma, se viven las circunstancias con el paciente a la vez que se están produciendo. Esta característica hace mucho más difícil la tarea de empatizar y a la vez adoptar una distancia necesaria y adecuada que permita la contención de las ansiedades puestas en escena. Es frecuente que la situación de bloqueo emocional que presentan algunos pacientes en estas circunstancias extremas, o de terror sin nombre, produzcan en los profesionales tratantes un alejamiento de su estado emocional, sin poder leer lo que este transmite. En este sentido, será tarea del psicólogo ayudar a dar un sentido a ese estado emocional del paciente para que este pueda ser entendido por el resto de profesionales, de forma que no se produzca una disociación entre su estado mental y físico. Por otra parte, estas situaciones pueden hacer oscilar al profesional entre la omnipotencia, fruto del sentirnos diferentes, alejados de las circunstancias del paciente y ejerciendo el rol de poder que puede representar la propia ayuda al paciente, y la impotencia, al conectar con nuestra propia debilidad por circunstancias vividas o anticipadas, similares a las que el paciente nos muestra. A la vez, vincularse con alguien que puede o va a morir, tampoco es tarea fácil. El peso de la realidad y la evolución de estos pacientes, a los que estamos tratando y con los que nos sentimos vinculados, nos sitúan en un punto importante de emocionalidad, necesario de elaborar, para proteger nuestra propia salud mental.

La ausencia de intimidad. Es habitual que las entrevistas psicológicas y posterior seguimiento se realicen en habitaciones que son compartidas por otros pacientes. Esta característica es radicalmente diferente a la que se produce en otros ámbitos de consulta psicológica. El paciente expone así su intimidad y el psicólogo también la suya propia, al mostrar su propia intervención y trato con el paciente. Es también muy frecuente la existencia de diferentes interrupciones, que se abra la puerta y que aparezcan otros profesionales con distintas finalidades. Esta situación requiere de una aproximación cuidadosa a los aspectos personales del paciente, intentando captar el momento idóneo para la exploración y respetando hasta dónde quiere expresar en ese momento determinado. Algunos gestos, como solicitar, siempre que sea posible, a otros profesionales que esperen, pedir a los acompañantes que salgan, preguntar si le viene bien hablar, poner la cortina separadora entre las camas, acercarse a la altura en la que esté el paciente o bajar el tono de voz, van a ser aspectos imprescindibles para que este pueda sentirse respetado en su intimidad.

La soledad en la tarea. Con mucha frecuencia, el psicólogo clínico que trabaja en el ámbito hospitalario no cuenta con otros compañeros que realicen la misma tarea en dicho ámbito y formen equipo clínico. Así como los médicos tienen otros médicos con competencias, tareas y dificultades similares de acuerdo a su especialidad, o enfermería cuenta con un cuerpo de enfermeros viviendo situaciones parecidas, no es esta la realidad para la mayoría de psicólogos. Por lo general, los psicólogos clínicos se encuentran adscritos a los servicios de psiquiatría, pero realizando la mayoría de ellos su función fundamentalmente en los centros de salud mental ambulatorios dependientes del mismo, por lo que los cometidos y población son muy diferentes. Esta circunstancia hace difícil compartir experiencias clínicas cotidianas y bajo una perspectiva similar en el ámbito hospitalario. Por otra parte, también otros psicólogos se encuentran ligados a otros servicios del Hospital (oncología, cardiología, neurología, digestivo...), con frecuencia de forma unipersonal, sin incluso conocerse entre ellos y sin posibilidad de relación, estando integrados además en un servicio en el que el objetivo prioritario compartido no es la salud mental del paciente. Esta situación de soledad y, por tanto, de fragilidad, choca con la expectativa de fortaleza que se le supone al psicólogo en su rol de soporte frente a estas situaciones emocio- 
nales tan complejas. No se tiene en cuenta, por tanto, el impacto emocional de las situaciones que se viven. Si ya es difícil compartir el dolor aún lo es más cuando no se comparten los mismos escenarios y perspectivas, percibiendo incluso una especie de incomodidad cuando se habla con otros profesionales sobre aquellas situaciones traumáticas que se habían delegado para su atención.

Las creencias erróneas. Una de las creencias con las que nos podemos encontrar en el ejercicio de nuestro trabajo es la consideración por parte de algunos profesionales sanitarios de que la incorporación de variables psicosociales hace perder rigor a las intervenciones con el paciente. En nuestra cultura actual, existe un ideal de funcionamiento sanitario, basado en la objetividad y en protocolos de actuación, considerando estos como garantía de una sanidad igualitaria y científica. Se puede considerar así, en algunos estamentos, que la introducción de lo psicológico y, por tanto, subjetivo, como un riesgo, más que como un requisito para el tratamiento y comprensión global del paciente. La consecuencia de actuar esta creencia de forma automática, muchas veces no pensada, sería la de prestar un segundo lugar a la atención psicológica o delegar esta de una forma global en los profesionales "psi". Sería por tanto un error considerar que toda la atención psicológica tiene que ser "suministrada" por psicólogos, sin reconocer que cada profesional, cuidador o miembro de la familia tiene una parte importante y complementaria en el cuidado psicológico del paciente ingresado. Las tareas de promover, formar y orientar en ese cuidado, así como la de detectar aspectos que lo estén impidiendo, con la finalidad de incidir en un posible cambio, se pueden considerar fundamentales entre las funciones del psicólogo clínico. Por otra parte, el temor de los propios psicólogos a no ser considerados en el mismo nivel de competencia de otros profesionales nos puede llevar a cierto mimetismo en el funcionamiento, con el riesgo de generalizar y argumentar nuestra intervención fundamental en base también a protocolos de actuación, en los que se pierda la dimensión individual y los significados propios del paciente en el marco de su proceso. La asunción, por tanto, de tareas que confrontan con los ideales colectivos, supone un elemento constante de dificultad en el trabajo cotidiano.

Frecuente imprecisión en los cometidos y tareas compartidas con otros profesionales (fundamentalmente de psiquiatría y enfermería en salud mental). El hecho de compartir un objetivo general común relativo a la salud mental, y también a menudo a los mismos pacientes, exigiría una exhaustiva definición de la intervención a realizar por parte de cada profesional, de forma que esta sea complementaria y beneficiosa, sin que se creen confusiones iatrogénicas. En este camino existe aún un recorrido importante de mejora. La elaboración de rivalidades personales y corporativas, que se despliegan con mayor fuerza en el ámbito público, es una condición ineludible para que la diferencia pueda ser permitida y tolerada. Aún sin intencionalidad, con frecuencia esta tarea es dificultada desde las propias instancias directivas, al priorizar o valorar determinadas intervenciones sobre otras, sin criterios objetivos que así lo justifiquen. Por otra parte, algunas de dichas rivalidades forman parte de la misma dimensión: para unos, los psicólogos clínicos, sería asimilar que otros, los psiquiatras, estaban primero en el ámbito sanitario, permitiendo así reconocer el recorrido realizado y la experiencia previa; para otros, los psiquiatras, sería hacer hueco a los ya no tan recién llegados, pudiendo valorar también su perspectiva y competencia. Al igual ocurriría entre psicólogos clínicos y enfermería en salud mental, que han sido los últimos en incorporarse. El resultado de elaborar lo anterior, y a veces se consigue, sería poder compartir lo que nos une, tolerar lo que admiramos y nos diferencia y trabajar de forma complementaria en la misma dirección.

Esta tarea de discriminación se debería realizar a dos niveles: el primero formaría parte de la definición de cada profesión, con sus estrategias, modalidades fundamentales de intervención y cometidos para los que fue contratada; y el segundo, de los equipos de trabajo, aplicando esos elementos generales definidos a cada caso concreto. Entre otras cosas, el marco general serviría para el establecimiento de qué profesional facultativo debería actuar como referente, en función de las demandas y necesidades prioritarias de intervención y a la vez, si interviene más de un profesional, con qué instrumentos complementarios cada uno de ellos.

La ausencia de psicólogos clínicos en las jefaturas. Esta situación dificulta la posibilidad de detección de necesidades psicológicas y de dar respuesta a las mismas con instrumentos y estrategias psicológicas. Por otra parte, representa además una carencia en la dirección y coordinación clínica de las intervenciones realizadas por los psicólogos, siendo esta asumida por médicos de otras especialidades, fundamentalmente psiquiatras, situación que nunca se da en sentido inverso. Si bien las actuales jefaturas ofrecen un soporte que es fundamental 
sobre aspectos que tienen que ver con la organización, con acuerdos entre servicios y con objetivos globales a conseguir, la diferencia de especialidad dificulta una visión más homogénea. Así, es frecuente que no se parta de un constructo común sobre prioridades, aspectos diagnósticos desde el ámbito psicológico, diseños y contenidos de la intervención psicológica específica, y sobre la comprensión global del paciente y familiares. La inercia de funcionamiento puede promover acciones centradas en el paciente, sin incluir la dimensión familiar y social y, como venimos diciendo, todo lo que tiene que ver con las dinámicas grupales que también inciden en la atención que se ofrece al paciente. Una consecuencia de responder desde modelos formativos no psicológicos sería la de administrar fármacos a pacientes que se podrían beneficiar óptimamente de una atención psicológica, a cambio de la infradotación de psicólogos para llevarla a cabo.

\section{Incorporación del Psicólogo Clínico al ámbito hospitalario}

Aunque el interés por la incorporación en el ámbito sanitario de las primeras promociones de psicólogos data de 1970 (Duro, 2016), esta se va produciendo lentamente y aún más la inclusión del psicólogo clínico en el ámbito hospitalario. Si bien es una realidad innegable, aún ahora su presencia es casi puntual, ya que es un facultativo que no estaba previsto originariamente en la constitución de los hospitales, mientras que sí lo estaban los médicos y enfermeros. En nuestro medio, en muchas ocasiones es una especialidad escasamente visible e identificada, sin representatividad en el organigrama organizativo de la mayoría de hospitales, debido, como hemos señalado anteriormente, a la carencia de unidades específicas, secciones o servicios de psicología clínica y con un número muy escaso de psicólogos clínicos contratados. Como consecuencia de lo anterior, es frecuente que exista un desconocimiento sobre su presencia, funciones, tareas o mecanismos de solicitud de intervención. Así, tanto médicos como enfermeros se enfrentan en su día a día con necesidades de pacientes y familiares, a las que no pueden dar repuesta desde modelos exclusivamente biomédicos, encontrándose sin herramientas y añadiendo por ello otro elemento de estrés en el desarrollo de su propio trabajo.

A pesar de que la inclusión del psicólogo clínico en el ámbito hospitalario genera un cambio de expectativas en el trabajo con el paciente y también un cambio o ajuste de roles por parte de otros profesionales, la tarea a realizar siempre ha de ser complementaria y colaborativa con dichos profesionales, aportando la visión específica de los procesos psicológicos observados. Esta tarea de colaborativa resulta aún más imprescindible y necesaria, cuando se trata de otros profesionales "psi", que realizan sus funciones en los hospitales.

Sería imposible trabajar si no se partiera del absoluto respeto hacia otras profesiones sanitarias y de la comprensión de otras formas de abordaje y prioridades a llevar a cabo con el paciente ingresado por situaciones agudas o enfermedades crónicas, en muchas ocasiones con importante riesgo vital. Establecer una complicidad con el profesional que realiza una demanda de intervención psicológica es totalmente necesario, en aras del cuidado del paciente y de los propios profesionales. Ser permeables a las necesidades y dificultades que plantean los profesionales sanitarios en su relación y cometidos con el paciente, intentando aportar claves desde este ámbito psicológico, será sin lugar a dudas una vertiente imprescindible de la intervención psicológica, creando a su vez una aproximación y cultura sobre los aspectos psicológicos.

Si bien la medicina más tradicional centra su atención en la intervención clínica con el paciente individual, en el ámbito hospitalario la presencia de familiares es prácticamente continua, siendo también la familia un elemento de apoyo y soporte de ciertas indicaciones médicas o todo lo contrario. Debido a esto, las familias no pueden ser ajenas o quedar excluidas de los propios procedimientos médicos.

El psicólogo clínico precisa contar, por tanto, de forma integrada, con una visión clínica, una visión social y una visión institucional aplicadas tanto al paciente, como a la familia y equipos institucionales. Esta visión tridimensional irrenunciable es la que va a permitir la realización de tareas globales de prevención, diagnóstico e intervención psicológica (Zas, 2011). Dicha intervención se aleja de la que se podría llevar a cabo en el ámbito privado, ya que la propia institución constituye un nivel imprescindible sobre el que se realiza la tarea (Bleger, 1994). Sin contar con esta visión integrada, la tarea será parcial y no estará contextualizada, perdiendo la riqueza comprensiva que aportan las variables mencionadas y repercutiendo en la intervención realizada. Por este motivo, si bien la presión provocada por la propia asistencia y también por el tiempo que los pacientes 
permanecen ingresados, definen abordajes, estilos y métodos con pacientes, familiares y equipos de salud, nunca se debe perder esa visión integral.

Para la realización de sus funciones, el psicólogo debe (Zas, 2011):

- Desarrollar habilidades especiales de comunicación y tener entrenamiento en comunicación en situaciones especiales.

- Conocer los principios y códigos éticos que regulan la práctica profesional propia y del resto de disciplinas con quienes interactúa.

- Saber planificar una intervención en relación a la demanda recibida, realizando una evaluación, una formulación de la situación y una selección de las estrategias que mejor se ajusten al problema y al resto de las características de la tarea.

- Conocer los límites de su intervención, en qué momento finalizar y cuándo colaborar con profesionales de otros servicios y especialidades que complementen la intervención aplicada.

- Realizar supervisión, dado que la interacción con pacientes y profesionales pone permanente en juego nuestra propia subjetividad, condicionando nuestra forma de pensar y sentir.

- Evaluar los resultados de sus intervenciones de modo que pueda hacer cambios durante el desarrollo de las mismas, así como conocer sus propias actitudes, prejuicios, limitaciones de conocimientos o habilidades.

- Desarrollar habilidades de gestión y negociación, al ocurrir la intervención psicológica en un contexto organizacional.

Junto a todo lo anterior, la responsabilidad en la formación en la docencia de residentes, específicamente de PIRes, pero también complementariamente de MIRes, será otra faceta imprescindible a desarrollar en el ámbito hospitalario.

\section{Aportaciones de la Psicología Clínica}

La modalidad de respuesta sanitaria a las necesidades planteadas por la población general se realiza, como no puede ser de otra manera, por las profesiones existentes en cada momento determinado.

A un nivel histórico, fue la medicina tradicional quien ocupó ese lugar, avanzando en la propia consideración de la salud en general, de la salud mental y en las estrategias de respuesta a dichas necesidades. El desarrollo de la medicina psicosomática y su interés por articular una vinculación entre el terreno de las ciencias biológicas y el de los significados (Parada, 2001), supuso un hito comprensivo que permitió un avance teórico y un cambio en la práctica clínica. La psicosomática sigue desarrollándose en la actualidad, como ciencia básica, como orientación médica y como práctica clínica (Álvarez, 2018). Actuales avances científicos ponen de manifiesto la influencia de factores psicosociales sobre la respuesta inmunológica, neurológica y endocrina (Ader, Cohen y Felten, 1991), destacando que el estrés, la ansiedad y la angustia son las condiciones psíquicas de mayor interrelación con los diversos sistemas del organismo, abriendo por ello la posibilidad de utilizar los tratamientos psicológicos para la mejora de dicho organismo en su conjunto. La consideración de que los acontecimientos a un nivel cualquiera de organización, desde el molecular al simbólico (Lipowsky, 1967), pueden repercutir y expresarse en los demás niveles obliga a un acercamiento diferente al paciente. Se introduce así la escucha, como una vía necesaria para la comprensión de esa subjetividad y se reconoce la influencia que tiene la relación establecida entre pacientes y profesionales en el curso de la enfermedad y eficacia del tratamiento (González de Rivera, 1999).

Esta perspectiva psicosomática ha ido permitiendo con el tiempo la incorporación de otros profesionales no médicos en la atención del paciente enfermo y junto a ella, la posibilidad de generar nuevas estrategias de intervención, entre ellas terapias psicológicas, con el objetivo de modular las respuestas emocionales o conductuales del paciente, así como mejorar su condición médica (Bayés, 1994; Berrocal, Fava y Sonino, 2016). Por otra parte, también es el acercamiento al paciente, contemplado desde su individualidad, conflictiva y trayectoria vital, una condición que permite detectar a aquellos pacientes en situación de mayor riesgo de desarrollar alteraciones psicológicas o psicopatológicas en el proceso de su enfermedad y también en sentido 
inverso. El inicio de la psiquiatría de enlace también tuvo sus orígenes en esta visión integral en la concepción del paciente.

La Organización Mundial de la Salud (OMS, 2001), propuso un modelo de salud mental que abarca, entre otros aspectos, el bienestar subjetivo, las percepciones de la propia eficacia, la autonomía, la competencia, la dependencia intergeneracional y la autorrealización de las capacidades intelectuales y emocionales de la persona. Este modelo pasa obligatoriamente por la implementación de estrategias de intervención que se alejan también de los modelos de la medicina tradicional.

La incorporación por tanto de las variables psíquicas y sociales, junto a las biológicas, implica para su consecución de la obligada cooperación entre diferentes especialistas. Desde esta perspectiva, la prevención de las enfermedades y la paliación del dolor y el sufrimiento adquieren una importancia fundamental.

En este sentido, la psicología clínica ha hecho aportes significativos, proporcionando modelos conceptuales explicativos de los trastornos mentales y del comportamiento y de los factores psicológicos concomitantes que presentan muchas enfermedades físicas. Junto a lo anterior, también ha proporcionado estrategias complementarias o alternativas para la prevención, evaluación, tratamiento y rehabilitación de estos trastornos, desde una concepción integral y multideterminada de la salud y enfermedad humanas (López Core, 2013, p.44).

Sin embargo, como acercamiento general, se ha entendido que la psicología de la salud se ocupa de las variables psicológicas que tienen consecuencias fisiológicas, mientras que la psicología clínica se ocupa de los problemas o trastornos psicológicos en sí mismos (Pérez, 1991). Ahora bien, cuando una persona presenta un sufrimiento y limitaciones en su funcionamiento vital, derivadas de dolencias físicas y/o psíquicas, inevitablemente interrelacionadas (Muñoz, 2012), requiere de una atención especializada global para la comprensión, manejo y afrontamiento psicológico (cognitivo, emocional, conductual), con la complejidad que hemos venido definiendo. Sería por tanto necesario aunar en un único campo teórico y asistencial la psicología clínica y de la salud, como concepción que evite el reduccionismo.

Como plantea Juan Carlos Duro (2016) en un alumbrador artículo sobre el estado de la psicología clínica en España: "La psicología clínica puede, en tanto disciplina novedosa, contribuir a la 'psicologización' de la atención a la salud/enfermedad, en el sentido de 'despatologizar' los problemas psicológicos (Costa y López, 2014), prevenir su aparición, ayudar a su superación y, en general, fomentar la salud en relación con cualquier aspecto del polo salud/enfermedad" (Duro, 2016, p.49).

A modo de referencia, podemos tomar la siguiente definición de competencias del psicólogo clínico y de la salud en el ámbito hospitalario: "La Psicología Clínica y de la Salud se ocupa del comportamiento humano, de los factores que lo determinan y de cómo inciden en la salud de las personas. Utiliza métodos que provienen de la psicología experimental para estudiar, explicar, prevenir, evaluar, diagnosticar, tratar y rehabilitar los aspectos psicológicos que afectan a la conducta, las emociones y las relaciones de las personas. Las intervenciones psicológicas tienen diversas modalidades y vías concretas de integración con los tratamientos médicos" (Grupo de trabajo de psicólogos en hospitales COP Madrid, 2005, p.6).

Ya en el año 1993 la OMS estableció una guía de tratamientos de eficacia probada, dedicando un amplio apartado a los tratamientos psicológicos. Desde entonces, ha sido imparable el avance en este campo todavía en constante desarrollo, habiéndose probado la eficacia de las intervenciones psicológicas individuales y grupales, para prevenir y compensar los efectos indeseables del proceso de enfermar (Andersen et al., 2004; Goodwin et al., 2001; Spiegel, 2001). En la actualidad se dispone de protocolos psicológicos de actuación en diversos ámbitos (oncología, cardiología, dolor, trasplantes, amputados, dermatología, obesidad, insuficiencia renal, neuropsicología, etc.) que permiten un abordaje científico y eficiente (Arranz, Barbero, Barreto y Bayés, 2003; Holland, 1999; Remor, Arranz y Ulla, 2003).

En las últimas décadas, las prestigiosas guías elaboradas por el National Institute of Clinical Excellence (NICE) vienen recomendando sistemáticamente las terapias psicológicas para un amplio rango de problemas de salud física (diabetes, esclerosis múltiple, enfermedades cardíacas...), ya que estos pacientes presentan riesgo elevado de sufrir ansiedad o depresión (NICE, 2009), siendo esta psicopatología de dos a tres veces más frecuentes en patologías orgánicas crónicas respecto a población sana (Moussavi et al. 2007). Aproximadamente entre el 20 y el $25 \%$ de los pacientes con enfermedades crónicas experimentan problemas psicológicos clínicamente significativos (White, 2001), sobre todo en grupos con enfermedades cardiovasculares, oncoló- 
gicas o neurológicas, cumpliéndose criterios de depresión mayor hasta en un 25\% de los casos (López Ibor, 2007). Así mismo, también en ciertos procesos agudos, se requiere una inmediata intervención en crisis (Grupo de trabajo de psicólogos en hospitales COP Madrid, 2005).

Pero, en un nivel de análisis más concreto, nos podemos preguntar cuáles pueden ser los beneficios específicos del abordaje psicológico en la patología orgánica: hay evidencia de que en personas con dolencias crónicas, el tratamiento psicológico puede mejorar notablemente la adherencia al tratamiento, el autocuidado y el llamado empoderamiento del paciente, involucrándose más proactivamente en su proceso de recuperación (NICE, 2009).

No reconocer y, por ende, no abordar, los aspectos psicológicos que influyen en los procesos salud-enfermedad, produce también un significativo impacto a nivel económico y social (London School of Economics, 2006; Radhakrishnan et al., 2013; Turpin, Richards, Hope y Duffy, 2008;Wahlbeck, 2011), en el incremento de la demanda asistencial, del consumo de fármacos, del tiempo de recuperación, de las visitas a urgencias y la insatisfacción de los pacientes (Carlson y Bultz, 2003; NICE, 2009).

En el Informe del Hastings Center (2005), elaborado por el centro de investigación bioética de Nueva York, sobre los fines de la medicina, se señala la importancia de atribuir a la promoción de la salud y prevención de la enfermedad el lugar prioritario que merecen. Esta idea incluye, necesariamente, la consideración de múltiples aspectos de índole psicológica. Como se expresa muy elocuentemente en el citado informe: "No se comprende que el temor a la mala salud, a la enfermedad y a su padecimiento puede provocar a menudo tanto sufrimiento como su existencia real. La amenaza que representa para alguien la posibilidad de padecer dolores, enfermedades o lesiones puede ser tan profunda que llegue a igualar los efectos reales que estos tendrían sobre su cuerpo" (The Hastings Center, 2005, p. 41), advirtiendo de que se atribuye al fármaco una tarea que correspondería a la psicoterapia y considerando que el sufrimiento mental que acompaña a la enfermedad no se suele detectar ni tratar adecuadamente.

Desde la clínica, se constata que la experiencia subjetiva de dolor y la evidencia de un mayor daño objetivable en el cuerpo no siempre están correlacionados, como muestran en muchos casos los resultados que ofrecen diferentes métodos diagnósticos que se emplean con los enfermos (pruebas de imagen, analíticas, etc.). Con frecuencia, dolor físico y psíquico se confunden y son expresados en los mismos términos, más aún en el ámbito hospitalario: el enfermo atribuye a los interlocutores una mayor competencia para ser entendido en los aspectos físicos y, por otra parte, el sistema sanitario resignifica el dolor y el sufrimiento psíquicos como enfermedad.

Así mismo, la respuesta psicológica a una enfermedad física puede consistir en una gama heterogénea de manifestaciones (miedo, desesperación, una profunda sensación de cansancio, ansiedad acerca del futuro, sentimiento de futilidad...), las cuales solo pueden ser debidamente atendidas mediante orientación/intervención psicológica.

Otro aspecto crucial a considerar explicitado en el mencionado trabajo, es el referente a aquellos pacientes (alrededor de un 50\% aproximadamente) que manifiestan lo que los autores denominan "padecimiento sin enfermedad", en relación a una serie de estados y experiencias de sufrimiento el cual no puede atribuirse únicamente a etiología fisiológica. En relación a esto, destaca especialmente el sufrimiento ocasionado por los problemas de salud mental, en sus diferentes niveles de gravedad e incapacidad, a pesar de la etiología funcional de la mayoría de ellos: "no todos los problemas de salud mental se originan a partir de una enfermedad, y resulta de suma importancia que para tomarlos en serio no sean necesarios unos fundamentos biológicos [...]. Un estereotipo difícil de erradicar es que la enfermedad mental es menos importante que la enfermedad física. De hecho, el padecimiento de un trastorno mental puede implicar el mismo grado de sufrimiento y discapacidad que los males de índole física" (The Hastings Center, 2005, pp. 41-42).

\section{Atención a la demanda de intervención psicológica}

La necesidad de atención psicológica hospitalaria puede ser detectada y tratada dentro del propio servicio de psiquiatría, o en el resto de servicios hospitalarios. Se delimita así a priori un perfil de pacientes de características diferentes en cuanto a su salud mental y condiciones físicas, que requieren intervenciones también 
diferentes. De igual manera, la relación del psicólogo clínico con los diferentes servicios hospitalarios (oncología, digestivo, paliativos, neurología, endocrino...), se va a definir de distinta manera si es un miembro que pertenece al mismo servicio en el que está ubicado el paciente, formulándose así una demanda intraequipo, consensuada dentro de ese equipo y para los pacientes de ese servicio, o si forma parte delservicio de psiquiatría. En este último caso, que es el mayoritario en la actualidad para los psicólogos clínicos, estos se integran en un equipo o unidad de interconsulta y enlace, al que llegan las derivaciones provenientes del resto de servicios. La intervención puede ir dirigida, por tanto, a:

\section{Pacientes}

- Enfermos con trastorno mental grave o con procesos agudos de enfermedad mental, que ingresan o reciben tratamiento ambulatorio en unidades dependientes del propio servicio de psiquiatría, como pueden ser las unidades de hospitalización breve, de transición hospitalaria, de trastornos de conducta alimentaria, adicciones, etc.

- Ingresados en diversos servicios hospitalarios, por motivos diferentes a su condición mental. Estos pacientes pueden tener previamente antecedentes en salud mental que requieran seguimiento o, aunque nos los tengan, sí se detecten en ese momento o pueden plantear sintomatología reactiva, en ocasiones severa, como fruto de la condición que provoca el ingreso. Una consideración especial merecen todos aquellos casos en los que puede concurrir un componente conversivo o facticio en la presentación del cuadro clínico, precisándose tanto de un diagnóstico adecuado como de un tratamiento acorde al mismo. Asimismo, tanto los pacientes que sufren pérdidas de gran impacto, con riesgo de evolucionar hacia duelos patológicos u otras alteraciones, como aquellos en los que se detecta un componente psicosomático en el inicio o curso de la enfermedad, van a ser susceptibles de intervención psicológica.

- Ambulatorios, derivados desde diferentes servicios a las consultas externas de psicología clínica, dependientes habitualmente del servicio de psiquiatría poniendo el foco en aspectos relacionados con la salud o determinada situación traumática severa, bien con una perspectiva preventiva y de promoción de salud o clínica propiamente dicha. Las modalidades de intervención pueden ser tanto individuales como grupales, dependiendo del perfil de pacientes y de los acuerdos entre servicios. Aunque no siempre, en bastantes casos la consulta ambulatoria supone la continuación de una intervención iniciada durante el ingreso, siendo una de las tareas discriminar de forma adecuada qué pacientes requieren derivación posterior al centro de salud mental que les corresponda.

- Ingresados o ambulatorios, que precisan diagnósticos diferenciales a nivel neuropsicológico o evaluaciones previas a determinados procedimientos médicos (trasplantes, cirugías...).

\section{Profesionales}

Los profesionales sanitarios pueden verse necesitados de asesoramiento sobre cómo abordar y manejar complejas situaciones, en las que los procesos psicoemocionales pueden desempeñar un papel nuclear y que trascienden el conocimiento y el saber hacer de sus respectivos campos de especialización. Se pueden encontrar, de este modo, desprovistos de herramientas para el manejo efectivo de estas situaciones y sin embargo, solicitar intervenciones centradas en el paciente, aun cuando este no lo desee, cuando quien tiene la dificultad para el manejo son el propio médico o enfermero. Este trabajo de "reconversión" de la demanda, ofreciendo al profesional la ayuda que precisa irá generando una mayor capacitación en el desarrollo de habilidades en su relación con el paciente y en la atención a sus aspectos psicológicos.

No podemos olvidar que, al igual que ocurre en otros contextos, cualquier intervención psicológica requiere del acuerdo y necesidad de aquel a quien se dirige la intervención, no pudiéndose realizar sin que este lo desee o sin que se dé cuenta (Utrilla, 1998). Podría ser el caso de muchos pacientes "difíciles", que plantean serios problemas relacionales o de seguimiento de pautas terapéuticas, pero para nada desean hablar con un psicólogo. 
En estos casos, no es que no esté indicada una intervención, que sin duda puede estarlo, pero lo que habrá que clarificar es con quien se realiza dicha intervención. En este caso, sería presumiblemente con el médico o enfermero que son quienes muestran la dificultad o preocupación.

Un primer nivel será, por tanto, esclarecer quién precisa la ayuda. El desarrollo de este nivel de intervención, que tiene que ver con clarificar la demanda de los propios profesionales, se puede ir realizando a un nivel cotidiano, trabajando cada derivación y alcance de la misma, y también a otro nivel, que sería el de ir creando un lenguaje y comprensión psicológica de los pacientes y situaciones, a través de la coordinación constante y de las diferentes acciones formativas o sesiones clínicas que se pueden realizar no solo con profesionales concretos, sino también con equipos. Salir de ese marco de intervención centrada en el paciente, a otro más general que incluya las necesidades de los profesionales requiere de una alianza constante, fruto del trabajo en equipo.

Una realidad que se observa es que a mayor sensibilidad frente a lo psicológico, también hay una mayor demanda formativa y de espacios de reflexión de equipos, que además de un beneficio para los pacientes, representan un cuidado de la salud mental de los propios profesionales.

La mayor capacitación de los profesionales en competencias para la comprensión y manejo de aspectos psicológicos reporta también una mayor seguridad en la relación con los pacientes y familiares, pudiendo actuar con mayor facilidad como continente de sus ansiedades y pudiendo comprender sus actuaciones y reacciones, no solo desde una clave de hostilidad, sino desde la subjetividad propia y la de los pacientes.

\section{Familias}

Debido a la mayor consideración que se ha ido dando a la participación de la familia en el proceso de bienestar del paciente enfermo, se está produciendo un cambio significativo en cuanto a la presencia de familiares en el ámbito hospitalario.

Fruto de lo anterior, cada vez existen menores restricciones para que el paciente pueda estar acompañado en cada momento. También se han abierto unidades que tradicionalmente habían estado más cerradas, siendo la convivencia con las familias una variable más de la dinámica hospitalaria. Prueba de ello son los tiempos establecidos para la información a familiares, la solicitud de su ayuda y cooperación en el cuidado del paciente y en fomentar la adhesión a los tratamientos, a la vez que aparecen anotaciones en las historias clínicas sobre el acompañamiento o no realizado por la familia.

Se considera, por tanto, que el apoyo emocional de la familia hacia el paciente ingresado es un elemento insustituible y esperanzador para el afrontamiento de la enfermedad y colaboración con los procedimientos médicos (Contreras y Palacios, 2013). Por otra parte, el ingreso de un paciente en el hospital conlleva también un impacto importante para la familia. Este impacto va a tener que ver con la propia estructura y funcionamiento familiar, la confluencia o no de otras problemáticas, su momento del ciclo vital y fortaleza de los vínculos familiares, así como del rol que el paciente ocupa dentro de esa familia (Plaszewska-Zywko y Gazda, 2012). Junto a lo anterior, el motivo del ingreso, recurrencia y pronóstico, así como las consecuencias derivadas de dicho ingreso, también van a constituir variables que ponen a prueba la fortaleza de la familia y la relación que esta pueda establecer con su familiar ingresado y con los profesionales. No podemos olvidar que los hospitales, como contexto organizativo, en su gran mayoría, están principalmente diseñados para facilitar el trabajo a los profesionales que desempeñan sus labores en ellos y no tanto para ayudar a las familias a adaptarse a un ambiente desconocido, el cual pueden percibir como hostil y amenazante, siendo todavía pocos los espacios en los que pueden expresar cómo están tramitando emocionalmente esta difícil experiencia (Davis, Kristjanson y Blight, 2003). Por ello, es fundamental que la familia sea considerada como una unidad básica de soporte, pero también receptora de ayuda y cuidado psicológico (Canga, 2014). En algunos momentos, es la angustia familiar la que no permite captar la información que los médicos les transmiten, teniendo la necesidad de ser también ellos escuchados y expresar libremente sus dificultades y temores. Asimilar y compartir la información recibida y contactar con los sentimientos que emergen a raíz de la misma, son procesos que en circunstancias muy traumáticas o extremas la familia no puede hacer sola, por lo que la intervención psicológica va a favorecer la propia estabilidad familiar, su capacidad en la toma de decisiones y su capacidad de afrontamiento ante circunstancias adversas. 
También existen circunstancias en que el trabajo con las familias es prioritario para los profesionales tratantes, y son todas aquellas en las que los pacientes, a consecuencia de su edad, situación física o mental, no se encuentran en condiciones de expresar su deseo o tomar decisiones importantes en relación con los procedimientos médicos.

La actitud de colaboración y de apoyo prestada por los médicos a las familias y su competencia y claridad junto al tiempo que dedican a responder a sus preguntas (Rodríguez Salvador, 2010), son elementos que contribuyen de forma importante a la percepción de apoyo y satisfacción de las propias familias, por lo que será importante colaborar en la capacitación para la adquisición de estas habilidades relacionales.

Una última consideración, sería la de tener en cuenta también la mayor exigencia que representa para los propios profesionales esa constante presencia de la familia.

Si bien una relación fluida de cooperación facilita el proceso de ingreso, en algunas ocasiones que habría que detectar, generalmente como fruto de su propio malestar o angustia, se convierte en un elemento de mayor control, observación y cuestionamiento de las actuaciones profesionales, representando un elemento de dificultad para los mismos y siendo precisa una intervención mediadora, que modifique en la medida de lo posible esa actitud o perspectiva.

\section{El programa de Interconsulta-Enlace: aspectos específicos}

\section{Equipo}

Las unidades de interconsulta-enlace, dependientes de los servicios de psiquiatría de los hospitales, están formadas en la actualidad de forma mayoritaria por médicos psiquiatras, y en segundo lugar por psicólogos clínicos y enfermeros especializados en salud mental. Conforman un equipo especializado para dar respuesta, como venimos diciendo, a las diferentes demandas relativas a la salud mental de pacientes pertenecientes a otros servicios del hospital, integrando diferentes estrategias farmacológicas, psicoterapéuticas y de cuidado, al servicio de esas necesidades y demandas que se planteen. Este equipo, se mueve en contextos cambiantes, relacionándose con un gran número de profesionales con conocimientos y culturas diferentes.

Esta característica obliga a mantener un grado de flexibilidad que facilite la creación de un lenguaje común relativo a la salud mental de los pacientes y permita la comunicación. En este sentido, las variables individuales y personales de estos profesionales también favorecerán o harán más dificultosas la realización de sus tareas en el amplio contexto hospitalario. Por otra parte, nunca puede faltar una mirada intraequipo, que reflexione sobre su propia capacidad de adaptación, tolerancia e integración de lo diferente, así como sobre la presencia de otros fenómenos primarios que puedan dificultar la colaboración y generar intervenciones paradójicas en su ámbito de actuación.

\section{Ámbitos}

Las tareas y actividades de interconsulta y enlace son necesarias y complementarias, presentando algunos aspectos comunes y otros específicos. Se sustentan en el ofrecimiento de una perspectiva o formación en el ámbito de la salud mental, que no tiene el médico derivante, o en la que se considera no competente. En ambos casos, el proceso de intervención incluye (Levenson, 2006) hablar con el derivante, revisar la historia clínica actual y pasada del paciente, entrevistar al paciente y/o familiares, formular estrategias diagnósticas y terapéuticas, devolver la información al médico derivante y ofrecer seguimiento periódico, si se precisa.

Será adecuado dar respuesta a una demanda a través de una tarea de interconsulta, cuando lo que se precisa es una intervención puntual y circunscrita al momento en que se encuentra el paciente durante su ingreso. La tarea de enlace, sin embargo, implica un mayor grado de colaboración y puede extenderse más allá del ingreso del paciente. Es la colaboración que se establece con el servicio que ha solicitado la intervención (médicos, enfermeros) proporcionando información acerca de los procesos psíquicos del enfermo y su familia, incluyen- 
do sugerencias de manejo, interpretación o mediación, con el objeto de suprimir conflictos, si existen, entre el paciente y el equipo asistencial (Zas, 2011). Esta tarea supone también la unión de grupos profesionales diferentes con objeto de docencia e investigación conjunta.

Una de las tareas más estructuradas dentro del trabajo de enlace, es la denominada como programas de enlace que prolonga la atención psicológica prestada, más allá de la etapa de ingreso, extendiéndose también a nivel ambulatorio. Por precisar de un acuerdo de colaboración entre servicios, deben estar avalados desde las jefaturas y contar al menos con un profesional responsable del programa en cada uno de los servicios, encargándose de la implementación y desarrollo de los mismos. Estos profesionales serán los encargados de promover y cuidar el funcionamiento y cumplimiento de objetivos, así como de evaluar los resultados, sugiriendo elementos de actualización y mejora. Para que sean efectivos deben contar con un adecuado reparto de responsabilidades y compromisos de actuación definidos y evaluables.

En el establecimiento de qué programas concretos se ponen en marcha, pueden estar presentes determinados requerimientos por parte de la propia administración y de las políticas sanitarias del momento, demandas sociales apremiantes, prioridades establecidas en el ámbito del propio hospital o del propio servicio de psiquiatría, así como una mayor sensibilización de determinados servicios hacia los aspectos psicológicos, que puede actuar como un motor de demanda.

En su dimensión práctica, para que estos programas puedan funcionar adecuadamente, es preciso que se difunda su existencia entre los profesionales implicados en el mismo. De la misma manera, se debe garantizar que también sean conocidos los términos de funcionamiento, los requisitos de inclusión de pacientes y los mecanismos de activación de las derivaciones, incluyendo quien las realiza y quien las recepciona. Si bien esto parece una obviedad, a veces no resulta fácil llevarlo a la práctica, dada la gran movilidad de profesionales (residentes, profesionales interinos, suplentes, turnicidad, etc.), que ocupan un lugar en este engranaje, sin que por la rapidez y sobrecarga del funcionamiento hospitalario, hayan tenido oportunidad de acceder a los términos del programa o a su propia existencia.

\section{Modalidades de Intervención psicológica en Interconsulta-Enlace}

Toda intervención psicoterapéutica, más allá de cualquier orientación o modelo de partida, precisa contemplar la subjetividad del paciente enfermo, distanciándose así del a priori modelo de funcionamiento médico, basado en conocimientos y saberes "objetivos". No podemos olvidar que estar enfermo, sentir dolor o anticipar la muerte, varía de forma considerable de unos pacientes a otros, constituyendo ese núcleo de particularidad uno de los ejes para la intervención psicoterapéutica. Por otra parte, conocer las posibilidades de elaboración psíquica con las que cuenta un paciente determinado va a ser una condición primordial para la elección del tipo de terapia más adecuada (Utrilla, 1998). Así, consideramos que una oferta rígida, igual para todos y basada de forma exclusiva en protocolos, se torna claramente insuficiente, siendo fundamental establecer una "sintonía empática" (Borelle, 2018, p.10) con el paciente, acorde a su modalidad de funcionamiento mental.

Una de las cuestiones que suelen suscitar mayor duda en cuanto al manejo del sufrimiento psíquico, como expresión de la vida emocional interna del paciente enfermo (Mee, Bunney, Reist, Potkin y Bunney, 2006; Panksepp y Biven 2012) tiene que ver con la mayor o menor capacidad que tiene para la mentalización del mismo (Lecours, 2018). Cuando los componentes emocionales están más mentalizados, aparecerá en el paciente la tristeza adaptativa, propiamente dicha, que a su vez sirve para elaborar la propia situación, aunque puede ser muy dolorosa. En caso contrario, lo que aparecerá será la angustia, con escasas posibilidades de representación y que supera las posibilidades de regular el sufrimiento y, en situación muy prolongada de esta, la agonía (Lecours, 2018). En estos dos últimos casos, podemos observar una gradación en el desbordamiento del paciente, que puede llegar a desorganizarlo. Un conocimiento preciso del estado del paciente nos llevará a la adopción de determinadas estrategias de acompañamiento de su sufrimiento, pero teniendo siempre como objetivo, más que el que desaparezca, sí el hacerlo más tolerable.

Valorar esta condición permite que la tarea terapéutica se adecúe a las características del paciente y no solo quede al servicio de intereses burocráticos. Siendo coherentes, el ideal terapéutico que puede ofrecer un de- 
terminado modelo para el profesional, siempre debe ser contrastado, por tanto, a la luz de las características subjetivas de cada paciente o grupo.

Por otra parte, al igual que la confianza en el médico tratante supone un elemento primordial en el tratamiento del paciente, también la eficacia terapéutica está determinada por la sensación de continuidad que la intervención psicoterapéutica debería proporcionarle a lo largo de su ingreso y/o enfermedad. Si el sistema motivacional de hetero-autoconservación, amenazado en este tipo de pacientes, se construye evolutivamente con alguien que cuida y sostiene (Bleichmar, 1997), esta presencia terapéutica, acompañante y cuidadora, tiene un efecto contenedor frente a las angustias más desbordantes. Durante el ingreso hospitalario, esta sensación de continuidad se realiza en sesiones más cortas y frecuentes, incluso diarias en función de las necesidades, sintiéndose el paciente en la mente de alguien que le acompaña en su padecimiento.

Al igual que en otros contextos, también en el ámbito hospitalario las modalidades de intervención pueden ser variadas, si bien todas deben tener contemplado un momento de inicio y un final, ya que es en otro ámbito, el de los centros de salud mental, donde son tratados los pacientes con psicopatología a largo plazo. Las propias características de los pacientes y contexto, favorecen las intervenciones en crisis, de resolución de problemas, psicoterapia breve de apoyo, acompañamiento terapéutico y psicoeducativas. En todos los casos, además de favorecer la sensación de control de sí mismo, que con frecuencia pierde el paciente enfermo, también se intentará contribuir a que pueda continuar con sus proyectos vitales, manteniendo la enfermedad en el lugar que corresponde dentro de su biografía global (Navarro Góngora, 2009), sin que el rol de enfermo defina completamente su identidad. Para ello, el terapeuta deberá poner especial interés en facilitar la comunicación y dirigir la exploración de temas relevantes, que puedan ser tratados en el tiempo del que se dispone y que sean apaciguadores para el paciente en su condición de enfermo.

Estas intervenciones pueden ser realizadas en diferentes marcos:

- Individual: poniendo su mirada comprensiva en diferentes aspectos según la formación de partida del terapeuta, pero con el foco en la identificación de aspectos que afectan a la adaptación y afrontamiento de la enfermedad, preocupaciones en torno a la misma y consecuencias derivadas, incluido el cuidado personal en el más amplio sentido. Si bien los tratamientos de larga duración, para pacientes con diagnósticos psicopatológicos, como acabamos de nombrar, están reservados al ámbito de los centros de salud mental, en algunos casos, por las características de determinados ingresos, a veces los seguimientos psicológicos se prolongan también durante periodos muy prolongados, permitiendo lógicamente un grado de profundización mayor en variables personales y biográficas, que cuando los ingresos son más breves.

- Grupal: reservadas habitualmente para perfiles de pacientes homogéneos en cuanto a la enfermedad o situación que motiva el ingreso. Pueden tener un matiz psicoeducativo o psicoterapéutico, adquiriendo la experiencia de compartir y el apoyo emocional que esto representa, una importante dimensión en la percepción, manejo y afrontamiento de la enfermedad. Se puede dirigir tanto a pacientes ingresados, ambulatorios o cuidadores y habitualmente representan un nivel importante de los programas de enlace.

\section{Docencia e Investigación}

Además de las funciones asistenciales, y al igual que el resto de facultativos sanitarios, los psicólogos clínicos están comprometidos con la función docente e investigadora, contemplando las dimensiones psicosomática y psicosocial como un eje vertebral de la misma. Participar y diseñar diversas acciones y programas formativos será por tanto una tarea fundamental del psicólogo clínico (Real Decreto 2490/1998, 1998).

Asistencia, docencia e investigación, deben estar presentes de forma conjunta y promoviendo líneas de investigación a partir de la práctica clínica, que ayuden en la mejora de los procesos implicados y puedan ser utilizados para un mejor abordaje de los enfermos. Estas funciones pueden realizarse dentro del propio servicio de psiquiatría o en otros servicios y se pueden contemplar a tres niveles: 
1. Formación de Pires. La modalidad formativa de la especialidad, a través de la vía de residencia, implica la rotación obligatoria por interconsulta-enlace, como una de las que se realizan en el ámbito hospitalario. En esta unidad, además de participar en todas las tareas asistenciales, es preciso que el residente cuente con los espacios necesarios de docencia y supervisión que aporten coherencia comprensiva al conjunto de las intervenciones. La docencia debe contemplar todas las áreas en las que, según hemos ido nombrando, va a tener que desarrollar sus funciones: pacientes, familiares y profesionales. En este sentido, conocer el impacto de la enfermedad en un sentido amplio, los métodos de evaluación e intervención sobre los sistemas de apoyo y fuentes de estrés, incluido el contex to hospitalario, será un eje fundamental de la docencia. Junto a lo anterior, el residente tendrá que diferenciar las reacciones normales y patológicas en el proceso de enfermedad. En este sentido deberá aprendera detectar y derivar a otros profesionales, fundamentalmente psiquiatras, los casos en que la psicopatología del paciente o su gravedad sintomática requieran tratamiento farmacológico. Entre estos casos, se incluyen también aquellos trastornos psicopatológicos que son producidos por determinadas alteraciones médicas o farmacológicas relacionadas con la propia salud del paciente ingresado.

De forma complementaria, la docencia incluirá el diseño de las intervenciones más adecuadas para un determinado perfil de pacientes en cada fase de la enfermedad y según el pronóstico de la misma, así como aspectos relativos a la relación entre pacientes, familiares y profesionales, que influyen en el bienestar de los mismos y en el curso de las enfermedades. Una consideración indispensable será la de transmitir a los Pires que su formación, bagaje personal y capacidad de contención van a representar el medio primordial para que puedan realizar su función como clínicos. Para ello, deben ofertarse espacios de intercambio y supervisión, en los que puedan expresar ciertas emociones emergentes en relación a sus experiencias, considerando esto un elemento diferenciador de calidad y de cuidado del propio residente.

2. Colaboración en la formación de médicos, enfermeros y residentes (del propio servicio de psiquiatría y/o de otros servicios hospitalarios) sobre aspectos psicológicos y diferentes problemáticas en la relación con los pacientes o familiares. Esta formación contribuye al estrechamiento de la cooperación entre servicios, a la vez que va configurando una comprensión integral de la salud, en la que los aspectos psicológicos van estando presentes, de forma progresiva, en las propias intervenciones clínicas. Así, la participación en sesiones clínicas o de actualización sobre diferentes temáticas, pueden formar parte de las actividades habituales. Por otra parte, es importante considerar que la mayor capacitación que adquieren los profesionales en los aspectos psicológicos, ofrece también una mejora en el trato con el paciente y en la percepción subjetiva de la atención que ofertan y su propia satisfacción en el rol que realizan.

3. Alumnos de psicología, tanto de grado como de postgrado, en el caso de hospitales que han realizado convenios de colaboración para la formación de alumnos en prácticas, a los que se les debe transmitir de forma global los conocimientos que avalan las diferentes intervenciones psicológicas que observan durante su periodo formativo.

\section{Consideración final}

"Uno no sabe nunca, si lo que ha escrito entraña algún valor, y menos aún si podrá escribir algo valioso en un futuro". M. Vargas Llosa

A modo de síntesis, solamente resaltar que la psicología clínica y de la salud cuenta con un importante marco teórico y conceptual para definir el alcance de la intervención psicológica en el ámbito hospitalario. Debido a ello, una buena práctica no debería circunscribirse, en ningún caso, solamente al paciente, dejando sin con- 
templar todos los ámbitos que hemos ido mencionando, incluida la dimensión institucional y lo relativo a los profesionales y familiares implicados.

\section{Referencias}

Ader, R., Cohen, N. y Felten, D. L. (1991). Psychoneuroimmunology (2th Edition). Nueva York, Estados Unidos: New York Press.

Álvarez, M. (2018). Psicosomática para todos [Editorial]. Psicosomática y Psiquiatría, 6, 17-20.

Andersen, B., Farrar, W., Golden-Kreutz, D., Glaser, R., Emery, C., Crespin, T..., Carson, W. E. (2004). Psychological, behavioural and immune changes after a psychological intervention: a clinical trial. Journal of Clinical Oncology, 22, 3570-3580. https://doi.org/10.1200/JCO.2004.06.030

Arranz, P., Barbero, J., Barreto, P. y Bayés, R. (2003). Intervención emocional en cuidados paliativos. Modelo y protocolos. Barcelona, España: Ariel.

Bayés, R. (1994). Psiconeuroinmunología, salud y enfermedad. Cuadernos de Medicina Psicosomática, 30, 28-34.

Berrocal, C., Fava, G. A. y Sonino, N. (2016). Contribuciones de la Medicina Psicosomática a la Medicina Clínica y Preventiva. Anales de Psicología,32, 828-836. https://doi.org/10.6018/analesps.32.3.219801

Bleichmar, H. (1997). Avances en psicoterapia psicoanalítica. Barcelona, España: Paidós.

Bion, W. R. (1974). Experiencias en grupos. Barcelona, España: Paidós.

Bleger, J. (1994). Psicohigiene y psicología institución. Buenos Aires, Argentina: Paidós. (Obra original publicada en 1966).

Borelle, A. (2018). La mentalización en el proceso clínico de pacientes con enfermedades somáticas. Instrumentos de evaluación. Mentalización. Revista de Psicoanálisis y Psicoterapia, 10, 1-27. Recuperado de https://revistamentalizacion.com/ultimonumero/abril borelle.pdf.

Canga A. (2014). Sobre los cuidadores de personas dependientes. Anales del Sistema Sanitario de Navarra, 37, 147-150. https://doi.org/10.4321/S1137-66272014000100018

Carlson, L. y Bultz, B. (2003). Benefits of psychosocial oncology care: improved quality of life and medical cost offset. Health and Quality of Life Outcomes, 1(8). Recuperado de http://www.hqlo.com/content/1/1/8. https://doi.org/10.1186/1477-7525-1-8

Contreras, A. M. y Palacios, X. (2014). Contribuciones de la Psicología al manejo interdisciplinario del paciente en Unidad de Cuidados Intensivos (UCI). Revista de la Universidad Industrial de Santander. Salud, 46(1), 47-60. Recuperado de http://www.scielo.org.co/scielo.php?script=sci arttext\&pi$\mathrm{d}=$ S0121-08072014000100006\&lng=en\&tlng=es

Costa, M. y López, E. (2014). Los problemas psicológicos no son enfermedades. Madrid, España: Pirámide.

Davis, S., Kristjanson, L. J. y Blight, J. (2003). Communicating with families of patients in an Acute Hospital with Advanced Cancer. Cancer Nursing, 26, 337-345. https://doi.org/10.1097/00002820-200310000-00001

Donker, F. J. (1991). Medicina Conductual y Psicología de la Salud. En G. Buela y V. E. Caballo (Eds), Manual de técnicas de terapia y modificación de conducta y salud. Madrid, España: Siglo XXI.

Duro, J. C. (2016). La Psicología Clínica en la sanidad pública en la Comunidad de Madrid: la insuficiente consolidación de una profesión sanitaria.Clínica y Salud,27, 45-50. https://doi.org/10.1016/j.clysa.2016.05.001

Gómez, R. (2004). El estrés laboral del médico: burnout y trabajo en equipo. Revista de la Asociación Española de Neuropsiquiatría, 24, 3104-3116.

González de Rivera, J. L. (1999). El desarrollo histórico de la Consulta Psiquiátrica en el Hospital General: De la Medicina Psicosomática a la Psiquiatría de Enlace. Psiquis, 21(1), 1-16.

Goodwin, P. J., Leszcz, M., Ennis, M., Koopmans, J., Vincent, L., Guther, H..., Hunter, J. (2001). The effect of group psychosocial support on survival in metastatic breast cancer. The New England Journal of Medicine, 345, 1719-1726. https://doi.org/10.1056/NEJMoa011871

Groves, M. S. y Muskin, P. R. (2006). Tratado de Medicina Psicosomática (Tomo I). Barcelona, España: Ars Médica. 
Grupo de Trabajo de Psicólogos en Hospitales COP Madrid (2005). Planificación y estrategia para la creación y puesta en marcha de un servicio de psicología clínica y de la salud. Recuperado de http://www.copmadrid. org/pdf/hospitales.pdf

Heyland, D. K., Rocker, G. M., Dodek, P. M. y Kutsogiannis, D. J. (2002). Family satisfaction with care in the intensive care unit:results of a multiple center study. Critical Care Medicine, 30, 1413-1418. https://doi. org/10.1097/00003246-200207000-00002

Holland, J. (1999). Update: NCCN Practice Guidelines for the management of psychosocial distress. Oncology, 13, 459-507.

Kaës, R. (1989). La institución y las instituciones: estudios psicoanalíticos. Buenos Aires, Argentina: Paidós. Kaminsky, G. (1990). Dispositivos institucionales. Buenos Aires, Argentina: Editorial Lugar.

Lecours, S. (2018). Niveles de mentalización del sufrimiento en la clínica: Agonía, desamparo y tristeza adaptativa. Mentalización. Revista de psicoanálisis y psicoterapia, 11. Recuperado de https://revistamentalizacion.com/ultimonumero/oct lecours.pdf

Levenson, J. L. (2006). Tratado de Medicina psicosomática (Tomo I). Barcelona, España: Ed. Ars Médica.

Lipowsky, Z. J. (1967). Review of consultation psychiatry and psychosomatic medicine: clinical aspects. Psychosomatic Medicine, 29, 201-224. https://doi.org/10.1097/00006842-196705000-00001

López Core, R. (2013). Desarrollo de la especialidad de Psicología Clínica en Costa Rica: Perspectivas futuras. Revista Costarricense de Psicología, 32(1), 43-60.

Moussavi, S., Chatterji, S., Verdes, E., Tandon, A., Patel, V., Ustun, B. (2007). Depression, chronic disease and decrements in health. Results from the World Health Surveys. Lancet, 370, 851-858. https://doi.org/10.1016/ S0140-6736(07)61415-9

National Collaborating Centre for Mental Health Commissioned by the National Institute for Health and Clinical Excellence (NICE) (octubre, 2009). Depression in adults with a chronic physical health problem: full guideline. Treatment and management. National Clinical Practice Guideline, 91. Recuperado de

http://www.nice.org.uk/nicemedia/pdf/CG91NICEGuideline.pdf

López Ibor, M. I. (2007). Ansiedad y depresión, reacciones emocionales frente a la enfermedad. Anales de Medicina Interna, 24(5), 209-211. Recuperado de http://scielo.isciii.es/scielo.php?script=sci arttext\&pi$\mathrm{d}=$ S0212-71992007000500001\&lng=es\&nrm=iso>. ISSN 0212-7199

London School of Economics and Political Science. Centre for Economic Performance. Mental Health Policy Group (2006). The depression report: a new deal for depression and anxiety disorders. Recuperado de http:// eprints.1se.ac.uk/archive/00000818

Mee, S., Bunney, B. G., Reist, C., Potkin, S. G. y Bunney, W. E. (2006). Psychological pain: A review of evidence. Journal of Psychiatric Research, 40, 680-690. https://doi.org/10.1016/j.jpsychires.2006.03.003

Muñoz, R. (2012). En busca de la integralidad: diez años de historia del departamento de salud mental de la Clínica Reina Sofía. Recuperado de http://psiquiatria.org.co/web/wp-content/uploads/2012/07/en-búsqueda-de-la-integralidad.pdf

Navarro Góngora, J. (2009). Terapia familiar con enfermos físicos crónicos. En J. Navarro Góngora y M. Beyebach (Comp.), Avances en terapia familiar sistémica (pp. 299-336). Barcelona, España: Ed. Paidós.

Organización Mundial de la Salud (OMS) (2001). Informe sobre la salud en el mundo. Salud mental: nuevos conocimientos, nuevas esperanzas. Ginebra, Suiza: Organización Mundial de la Salud.

Panksepp, J. y Biven, L. (2012). The archeology of mind: Neuroevolutionary origins of human emotion. Nueva York, Estados Unidos: Norton.

Parada, R. (2001). Patopsicología y Psicología en la Clínica Psiquiátrica. Santiago, Chile: Editorial Mediterráneo.

Pérez, M. (1991). Medicina, Psicología de la Salud y Psicología Clínica. Revista de Psicología de la Salud,1, 2144.

Plaszewska-Zywko, L. y Gazda, D. (2012). Emotional reactions and needs of family members of ICU patients. Anaesthesiology Intensive Therapy, 44(3), 145-149.

Radhakrishnan, M., Hammond, G., Jones, P. B., Watson, A., McMillian-Shields, F. y Lafortune, L. (2012). Cost of Improving Access to Psychological Therapies (IAPT) programme: An analysis of cost of session, 
treatment and recovery in selected Primary Care Trusts in the East of England region. Behaviour research and therapy, 51, 37-45. https://doi.org/10.1016/j.brat.2012.10.001.

Real Decreto 2490/1998 por el que se crea y regula el título oficial de psicólogo especialista en psicología clínica. Boletín Oficial del Estado (España), 288, de 2 de diciembre de 1998.

Remor, E., Arranz, P. y Ulla, S. (2003). El psicólogo en el ámbito hospitalario. Bilbao, España: Desclée de Brouwer.

Ricart, A. (2008). La comunicación en Medicina Intensiva. REMI: Libro Electrónico de Medicina Intensiva. Recuperado de http://www.medicina-intensiva-libro.com/2011/05/306-la-comunicacion-en-medicina.html

Rodríguez Salvador (2010). Comunicación clínica: cómo dar malas noticias. Recuperado de http://www.doctutor.es/wp-content/uploads/2010/03/Dar-Malas-Noticias-JJ-Rodriguez-S-2010.pdf

Spiegel, D. (2001). Mindmatters: grouptherapy and survival in breastcancer. The New England Journal of Medicine, 345, 1719-1720.

Rodríguez, J. y Zurriaga, R. (1997). Estrés, enfermedad, hospitalización. Granada, España: Escuela Andaluza de Salud Pública.

The Hastings Center (2005). Los fines de la Medicina. Barcelona, España: Fundació Víctor Grífols y Lucas. Recuperado de http://www.hospitalsantjoan.cat/wp-content/uploads/2018/09/fins medicina.pdf

Turpin, G., Richards, D., Hope, R. y Duffy, R. (2008). Delivering the IAPT programme. Healthcare Counselling and Psychotherapy Journal, 8(2), 2-8.

Utrilla, M. (1998). ¿Son posibles las terapias en Instituciones?: Estudio situacional. Madrid, España: Biblioteca Nueva.

Wahlbeck, K. (2011). European mental health policy should target everybody. European Journal of Public Health, 21, 551-553. https://doi.org/10.1093/eurpub/ckr122

White, C. A. (2001). Cognitive Behaviour Therapy for Chronic Medical Problems. A Guide to Assessment and Treatment in Practice. Nueva York, Estados Unidos: John Wiley \& Sons.

Zas, B. (2011). Experiencias en Psicología Hospitalaria. Recuperado de https://www.monografias.com/trabajos107/experiencias-psicologia-hospitalaria/experiencias-psicologia-hospitalaria.shtml

Artículo recibido: 03/02/2019

Artículo aceptado:19/02/2019 